\title{
Preparing for the cancer revolution
}

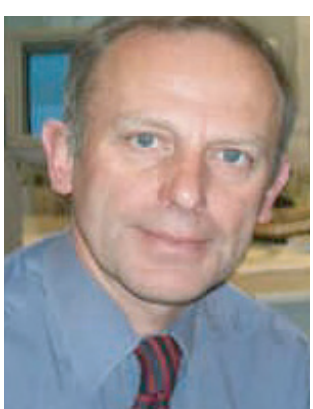

Karol Sikora

Professor of Cancer Medicine, Imperial College, Hammersmith Hospital and Scientific Director, Medical Solutions PLC, 27 Harley St, London W1G 8PZ, UK karolsikora@hotmail.com

\author{
'Increased consumerism in \\ medicine will lead to well-informed \\ and assertive patients seeking out \\ novel therapies and bypassing \\ traditional referral pathways \\ through global information \\ networks.'
}

The age of the world's population is rising dramatically. This will lead to a significant increase in the total burden of cancer with many patients living with considerable comorbidity. At the same time, new technology in many therapeutic areas is bringing improvements to the quality and length of life. Major advances in the following six endeavours are likely to have the greatest impact on cancer:

- Molecularly targeted drugs with associated sophisticated diagnostic systems to personalize care

- Biosensors to detect, monitor and correct abnormal physiology and to provide surrogate measurements of cancer risk

- Our ability to modify the human genome through systemically administered novel targeted vectors

- The continued miniaturization of surgical intervention through robotics, nanotechnology and precise imaging

- Computer driven interactive devices to help everyday living

- The use of virtual reality systems which, together with novel mood control drugs, creates an illusion of wellness

Over the last 10 years, a huge amount of fine detail on basic biological processes that become disturbed in cancer has been amassed. The key elements of growth factor binding, signal transduction, gene transcription control, cell cycle checkpoints, apoptosis and angiogenesis are now known. These have become fertile areas to hunt for rationally based anticancer drugs. This approach has already led to a record number of novel compounds currently in trials. Indeed, targeted drugs such as rituximab (Mabthera $\left.{ }^{\circledR}\right)$, trastuzumab (Herceptin ${ }^{\circledR}$ ), imatinib $\left(\right.$ Gleevec $\left.^{\circledR}\right)$, gefitinib (Iressa $\left.{ }^{\circledR}\right)$, bevacizumab (Avastin ${ }^{\mathrm{TM}}$ ), cetuximab (Erbitux ${ }^{\circledR}$ ) and erlotinib (Tarceva ${ }^{\circledR}$ ) are all firmly in routine clinical use. Over the next decade, there will clearly be a marked shift in the types of agents used in the systemic treatment of cancer.

As we know the precise targets of these new agents, there will be a revolution in how we prescribe cancer therapy. Instead of defining drugs for use empirically and relatively ineffectively for different types of cancer, we will identify a series of molecular lesions in tumor biopsies. Future patients will receive drugs that target these lesions directly. The human genome project provides a vast repository of comparative information on normal and malignant cells. The new therapies will be more selective, less toxic and administered for prolonged periods of time, and in some cases, for the rest of the patients life. This will lead to a radical overhaul of how cancer care is provided.

Investment in more sophisticated diagnostics is now urgently required. Holistic systems such as genomics, proteomics, metabolomics and methylomics provide fascinating clues as to where needles can be found in the haystack of disturbed growth. By developing simple, reproducible and inexpensive assays for specific biomarkers, a battery of companion diagnostics will emerge. It is likely that for the next decade these will be firmly rooted in tissue pathology, making today's histopathologist essential in moving this exciting field forward. Ultimately, the fusion of tissue analysis with imaging technologies may make virtual biopsies of any part of the body - normal and diseased, a possibility.

Individual cancer risk assessments will lead to tailored prevention messages and a specific screening program to pick up early cancer, and will have far reaching public health consequences. Cancer preventive drugs will be developed to reduce the risk of further genetic deterioration. The use of gene arrays to monitor serum for fragments of DNA containing defined mutations could ultimately develop into an implanted gene chip. When a significant 
mutation is detected, the chip would signal the holder's home computer and set in train a series of investigations based on the most likely type and site of the primary tumor.

There will be an increase in the total prevalence of cancer as a result of improved survival, as well as a change in cancer types to those such as prostate cancer, with longer survival. This will create new challenges in terms of assessing risks of recurrence, designing care pathways, use of IT and improving access to services. There will be new opportunities for further targeting and development of existing therapies as experience grows with risk factors over the longer term. Careful monitoring of patient experiences could help to improve results. Cancer could soon be a long-term management issue for many patients where they enjoy a high quality of life even with a degree of chronic illness.

The funding of cancer care will become a significant problem. Already we are seeing inequity in access to the taxanes for breast and ovarian cancer, and gemcitabine $\left(\mathrm{Gemzar}^{\circledR}\right)$ for lung and pancreatic cancer. These drugs are palliative, adding just a few months to life. The emerging compounds are likely to be far more successful, and their long-term administration making them considerably more expensive. Increased consumerism in medicine will lead to well-informed and assertive patients seeking out novel therapies and bypassing traditional referral pathways through global information networks. It is likely that integrated molecular solutions for cancer will develop, leading to far greater inequity than at present. Cost-effectiveness analyses will be used to scrutinize novel diagnostic technologies as well as therapies.

New financial structures will arise with consortia of the pharmaceutical, insurance and healthcare sectors, enabling future patients to choose the level of care they wish to pay for by insurance schemes or directly. By the year 2020, chemotherapy is likely to replace other treatment modalities for most cancers. Cancer will become a chronic, controllable illness rather like diabetes or hypertension today. Biomarkers of response will be used to guide, titrate and monitor chronic therapy. People living with cancer will receive care in an attractive hotel-like environment, rather than a hospital, run by competing private sector providers. Global franchises will emerge using the web to disseminate treatment plans and control their quality. State healthcare systems will become regulators and insurers, thus relinquishing their role as providers between the years 2005 and 2010. This transition will bring new ethical and moral dilemmas. Cancer's alternative futures will be created by the interaction of four complex factors: technological success, society's willingness to pay, future healthcare delivery systems and the financial mechanisms that underpin them.

\section{'New ethical and moral dilemmas will arise as we seek the holy grail of compressed morbidity. Living long and dying fast will become the mantra of 21 st century medicine.'}

Societal change could create a crisis in the provision of care. A decline in hierarchical religious structures, a reduction in family integrity through increasing divorce, greater international mobility and the increased selfishness of a consumer-driven culture will leave many patients lonely and with no psychological crutch to lean on at the onset of serious illness. There will be a global shortage of carers - the unskilled, low paid but essential component of any health delivery system. The richer parts of the world are now harnessing this from the poorer but eventually, the supply of this precious human capital will dry up. The ability of technology to improve health is assured. However, this will come at a price - the direct costs of providing it, and the care costs of the increasingly elderly population it will produce. We will simply run out of things to die from. New ethical and moral dilemmas will arise as we seek the holy grail of compressed morbidity. Living long and dying fast will become the mantra of $21 \mathrm{st}$ century medicine.

We welcome this timely and exciting new journal which will highlight many of the impending technological advances. Crossing the laboratory-clinical divide is never easy but a greater synergy has never more been needed. Please contribute articles that reflect the dawn of this new era of rational cancer treatment. 